P012

\title{
OPTIMAL TEXT-BACKGROUND LIGHTNESS COMBINATIONS OF TABLET DEVICES FOR VISUAL COMFORT UNDER A WIDE RANGE OF ILLUMINANCE LEVELS
}

\author{
Hsin-Pou Huang et al.
}

DOI 10.25039/x47.2020.PO12

\section{Paper accepted for the $5^{\text {th }}$ CIE Symposium on Colour and Visual Appearance}

The paper was selected by the International Scientific Committee (ISC) for presentation at the 5th CIE Symposium on Colour and Visual Appearance, Hong Kong, CN, April 21-22, 2020, which, due to the corona pandemic, could not take place. The paper has not been peer-reviewed by CIE.

\section{(C) CIE 2020}

All rights reserved. Unless otherwise specified, no part of this publication may be reproduced or utilized in any form or by any means, electronic or mechanical, including photocopying and microfilm, without permission in writing from CIE Central Bureau at the address below. Any mention of organizations or products does not imply endorsement by the CIE.

This paper is made available open access for individual use. However, in all other cases all rights are reserved unless explicit permission is sought from and given by the CIE.

CIE Central Bureau

Babenbergerstrasse 9

A-1010 Vienna

Austria

Tel.: +4317143187

e-mail: ciecb@cie.co.at

www.cie.co.at 
P012

\title{
OPTIMAL TEXT-BACKGROUND LIGHTNESS COMBINATIONS OF TABLET DEVICES FOR VISUAL COMFORT UNDER A WIDE RANGE OF ILLUMINANCE LEVELS
}

\author{
Huang, H.P. ${ }^{1}$, Wei, M. ${ }^{2, *}$, Ou, L.C. ${ }^{3}$ \\ ${ }^{1}$ Chihlee University of Technology, New Taipei City, CHINESE TAIPEI; ${ }^{2}$ The Hong Kong \\ Polytechnic University, Kowloon, HONG KONG; ${ }^{N}$ National Taiwan University of Science and \\ Technology, Taipei, CHINESE TAIPEI \\ minchen.wei@polyu.edu.hk
}

\begin{abstract}
The psychophysical experiment was carried out to investigate how illuminance level affected the visual comfort for e-reading on a tablet device. 44 Asian observers between 19 and 25 years of age (mean $=21.5, S D=1.2$ ) participated in the experiment, comprising 20 observers evaluated the visual comfort experiment under dark condition, $300 \mathrm{Ix}$ and $3000 \mathrm{Ix}$ and 24 observers evaluated the visual comfort experiment under $150 \mathrm{~lx}, 1500 \mathrm{~lx}$ and $15000 \mathrm{~lx}$. The experimental results show that the visual comfort interval scale below 1500 Ix conditions (i.e. Dark, $150 \mathrm{~lx}, 300 \mathrm{~lx}$ and $1500 \mathrm{~lx}$ ) were similar to each other, but not to the visual comfort interval scale above the $1500 \mathrm{~lx}$ conditions (i.e. $3000 \mathrm{~lx}$ and $15000 \mathrm{~lx}$ ). For the same lightness difference between text and background, the observers' judgements show that they felt more comfortable to read the document using white background than the document using black background under high illuminance level.
\end{abstract}

Keywords: Visual comfort, Tablet device, Illuminance level, Reading

\section{Introduction}

With the advances in mobile devices technology, e-reading on mobile devices is becoming more and more necessary in human's daily life. Human rely on e-reading devices to read news, get information, play games, and communicate with family and friends under different ambient lighting conditions, from lower illuminance level at night to higher illuminance level under daylight or from lower correlated colour temperature (CCT) level at warm lighting room to higher CCT level at cooler lighting room. Many mobile device manufacturers and studies consider how ambient lighting condition may affect the mobile interface design to promote user visual comfort and preference [Chen and Lin 2004, Lin and Huang 2006, Na and Suk 2015, Na and Suk 2016]. Our previous study investigated how ambient lighting condition affected the observers visual comfort for e-reading, and found that the visual comfort for e-reading the 20 text-background lightness combinations under different CCT levels were highly correlated. Comparing the results of the Pearson correlation coefficient between different CCT levels, these tendency of high correlation between different CCT levels does not appear to different illuminance levels. Our previous study showed that the Pearson correlation coefficient between the judgments under the dark surround and under the $3000 \mathrm{Ix}$ condition was 0.5 . However, the previous experiment was carried out with an ambient illuminance level below $3000 \mathrm{~lx}$ to investigate the visual comfort for e-reading in interior environments [Huang et al 2018]. With the above in mind, this study aimed to investigate how ambient illuminance level (including outdoor illuminance of $15000 \mathrm{~lx}$ ) affected the visual comfort for e-reading.

\section{Methods}

A paired comparison psychophysical experiment was carried out to investigate the visual comfort in a viewing booth, which was uniformly illuminated using either a 14-channel spectrally tunable LED device or a four-channel spectrally tunable LED device (ARRI SkyPanel S60-C) to produce light sources. The LED device was carefully adjusted to produce five light sources, comprising five levels of illuminance (i.e. $150 \mathrm{~lx}, 300 \mathrm{~lx}, 1500 \mathrm{~lx}, 3000 \mathrm{~lx}$ and $15000 \mathrm{~lx}$ ) with a horizontal CCT of $6500 \mathrm{~K}$, the relative spectral power distributions (SPDs) of these five light 
sources are shown in Figure 1 and Table 1 show the colorimetric characteristics of the ambient lighting conditions, measured using a calibrated JETI specbos 1211TM spectroradiometer. Apart from the five levels of illuminance, a dark condition was considered in this study. 44 Asian observers between 19 and 25 years of age (mean $=21.5$, SD $=1.2$ ) participated in the experiment, comprising 20 observers evaluated the visual comfort experiment under dark condition, 300 and $3000 \mathrm{Ix}$ and 24 observers evaluated the visual comfort experiment under $150 \mathrm{Ix}, 1500 \mathrm{~lx}$ and $15000 \mathrm{~lx}$. All observers had kept their chins being fixed on a rest during the experiment to ensure a similar viewing distance around $45 \mathrm{~cm}$ between the eyes and the iPad Air 2. An iPad Air 2 was placed on a $45^{\circ}$ viewing table which was placed at the center of the booth, as shown in Figure 2. For understanding the visual comfort of different textbackground lightness combinations, 20 text-background combinations were produced. The 20 text-background combinations were composed by all possible combinations of five achromatic colours, as listed in Table 2. Based on the 20 text-background combinations, 190 paired comparisons were generated, considering all possible paired comparisons of these 20 textbackground combinations. A total of 20 of the 190 paired comparisons were presented twice for testing the repeatability, meaning 210 paired comparisons in total were conducted by each observer under each surround. Each observer was asked to pick one that was more comfortable to read the text from the two text-background combinations, as shown in Figure 3. Thus, each observer made forced-choices for a total of 630 paired comparisons (210 paired comparisons in a random order $\times 3$ surrounds) in terms of visual comfort.

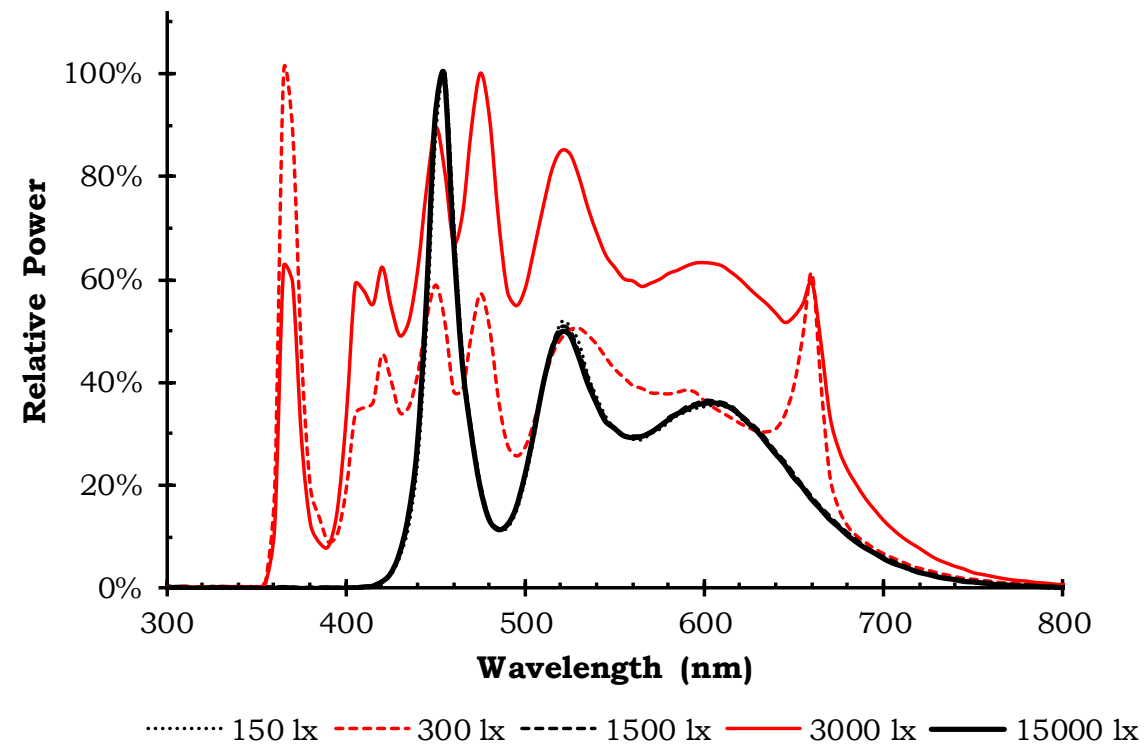

Figure 1 - The relative spectral power distributions (SPDs) of the light sources

Table 1 - Colorimetric characteristics of the light sources

\begin{tabular}{cccc}
\hline $\begin{array}{c}\text { Light source } \\
\text { Illuminance (1x) }\end{array}$ & $\mathbf{C C T}(\mathbf{K})$ & $\mathbf{C R I} \boldsymbol{R}_{\mathbf{a}}$ & $\boldsymbol{D}_{\mathbf{u v}}$ \\
\hline 150 & 6475 & 97 & 0.001 \\
300 & 6547 & 95 & 0.0039 \\
1500 & 6454 & 95 & -0.001 \\
3000 & 6533 & 98 & 0.0041 \\
15000 & 6471 & 98 & -0.002 \\
\hline
\end{tabular}




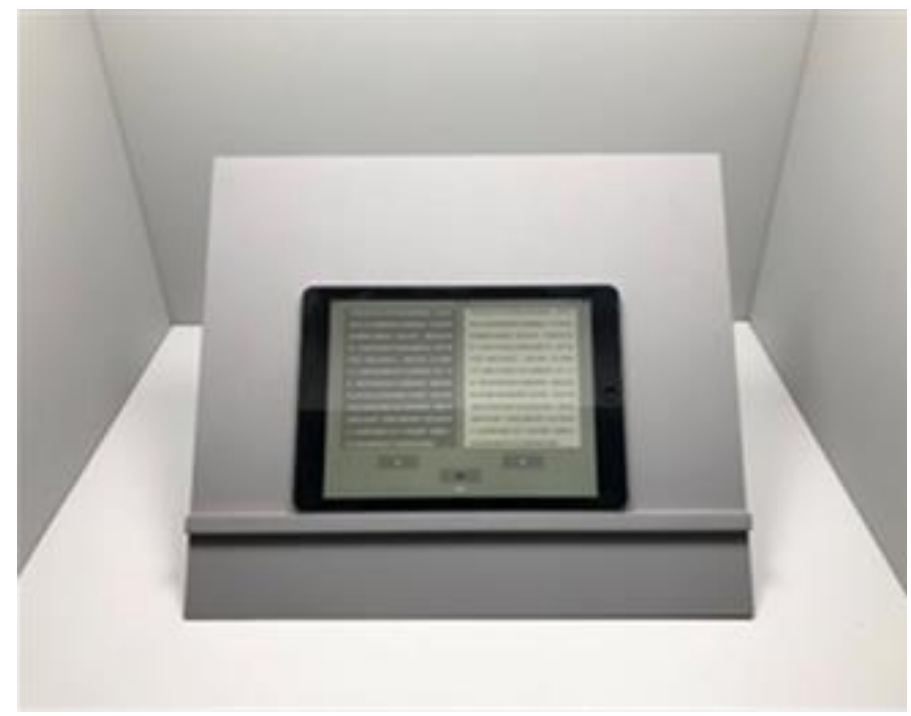

Figure 2 - The photograph of the experimental setup

Table 2 - Colorimetric characteristics of the five achromatic colours

\begin{tabular}{cccc}
\hline Achromatic color & $\begin{array}{c}\text { Luminance } \\
\text { Color }\end{array}$ & $\boldsymbol{L}^{*}$ & $\mathbf{( x , / \mathbf { m } ^ { 2 } \mathbf { ) }}$ \\
\hline Black & 0.7 & 1.6 & $(0.311,0.328)$ \\
Dark grey & 18.1 & 25.2 & $(0.307,0.327)$ \\
Medium grey & 77.3 & 50.9 & $(0.306,0.326)$ \\
Light grey & 196.5 & 75.3 & $(0.308,0.327)$ \\
White & 402.7 & 100.0 & $(0.307,0.326)$ \\
\hline
\end{tabular}

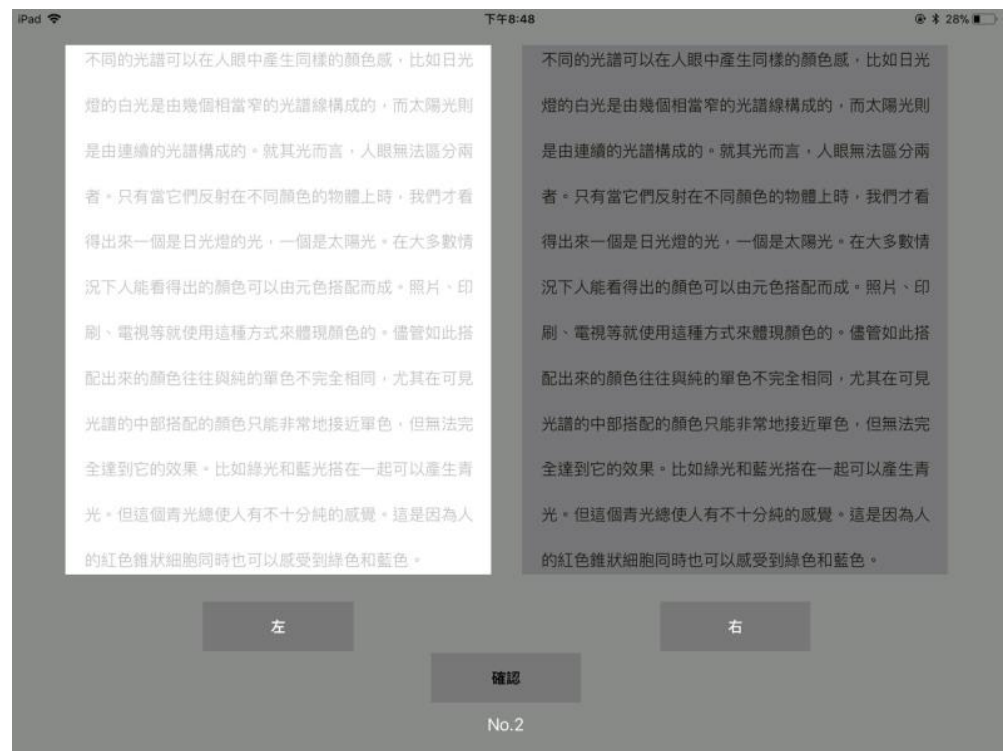

Figure 3 - A screenshot of the paired-comparison presented on the iPad Air 2 


\section{Results}

The repeatability test result for a total of 2640 paired comparisons presented twice shows that in general the observers made the same judgements for $84.4 \%$ of the replicated trials. This means that the psychophysical experiment was highly repeatable and stable for the 44 observers. The interval scales of visual comfort were determined by the results of paired comparison experiment using Thurstone case $V$ method [Thurstone1994]. The higher the interval scale, the more visual comfortable judged by the observers. When the ambient illuminance below $1500 \mathrm{Ix}$, the tendency of visual comfort interval scale did not seem to vary with the ambient illuminance. When the ambient illuminance above $1500 \mathrm{Ix}$, the tendency of visual comfort interval scale also did not seem to vary with the ambient illuminance. However, according to Pearson correlation coefficients (i.e., always below 0.7) between the judgments below those the $1500 \mathrm{~lx}$ conditions (i.e. Dark, $150 \mathrm{~lx}, 300 \mathrm{~lx}$ and $1500 \mathrm{~lx}$ ) and the judgments above those the $1500 \mathrm{Ix}$ conditions (i.e. $3000 \mathrm{Ix}$ and $15000 \mathrm{Ix}$ ), as shown in Table 3, it is appropriate to say that in general the impact of ambient illuminance on visual comfort was great, especially for the e-reading visual comfort between a dark surround and exterior illuminance levels.

Moreover, though the illuminance levels were found to have little impact on visual comfort for lightness difference, the observers' judgement scale did not always show the same tendency for the background colour. When the ambient illuminance below $1500 \mathrm{~lx}$, with the same lightness difference for text-background combinations, the visual comfort for the textbackground combinations with the black background were assessed more comfortable than the text-background combinations with the white background under lower illuminance level (i.e. Dark, $150 \mathrm{~lx}, 300 \mathrm{~lx}$ and $1500 \mathrm{~lx}$ ), as shown in Figure 4 (a)-(d). In contrast, with the same lightness difference for text-background combinations, the text-background combinations with the white background were assessed more comfortable than the text-background combinations with the black background under high illuminance level (i.e. $3000 \mathrm{~lx}$ and $15000 \mathrm{~lx}$ ), as shown in Figure $4(\mathrm{e})-(\mathrm{f})$.

Table 3 - Correlation between different illuminance levels

\begin{tabular}{cccccc}
\hline Correlation coefficient & & & & & \\
& Dark & $1501 \times$ & $3001 \times$ & $15001 \times$ & $30001 \times$ \\
\hline $1501 \times$ & 0.966 & & & & \\
$3001 \times$ & 0.820 & 0.715 & & & \\
$15001 \times$ & 0.954 & 0.906 & 0.918 & & \\
$30001 \times$ & 0.497 & 0.320 & 0.878 & 0.659 & \\
$150001 \times$ & 0.548 & 0.375 & 0.866 & 0.708 & 0.967 \\
\hline
\end{tabular}



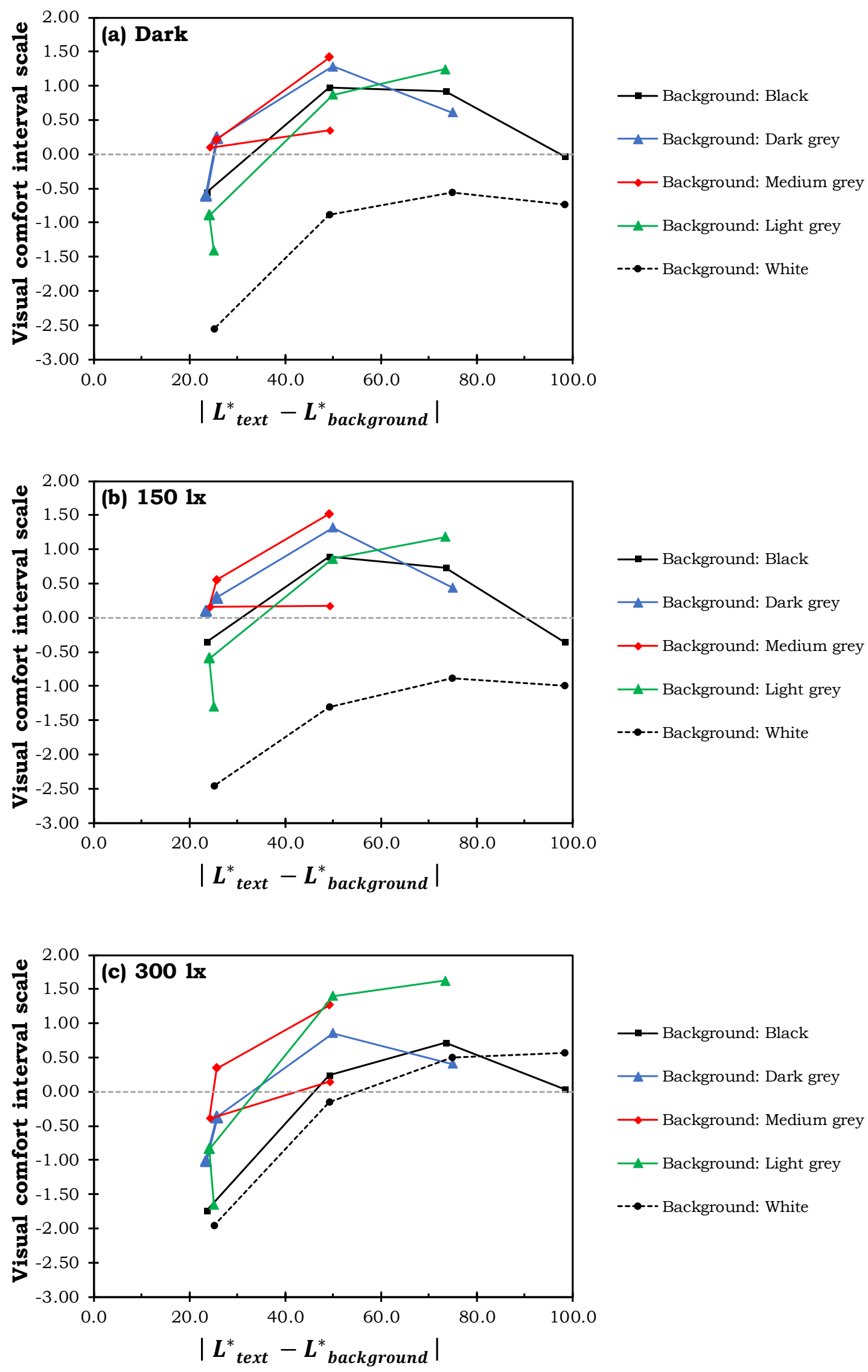

\footnotetext{
$\longrightarrow$ Background: Black

$\longrightarrow$ Background: Dark grey

$\multimap$ Background: Medium grey

$\longrightarrow$ Background: Light grey

---- Background: White
} 

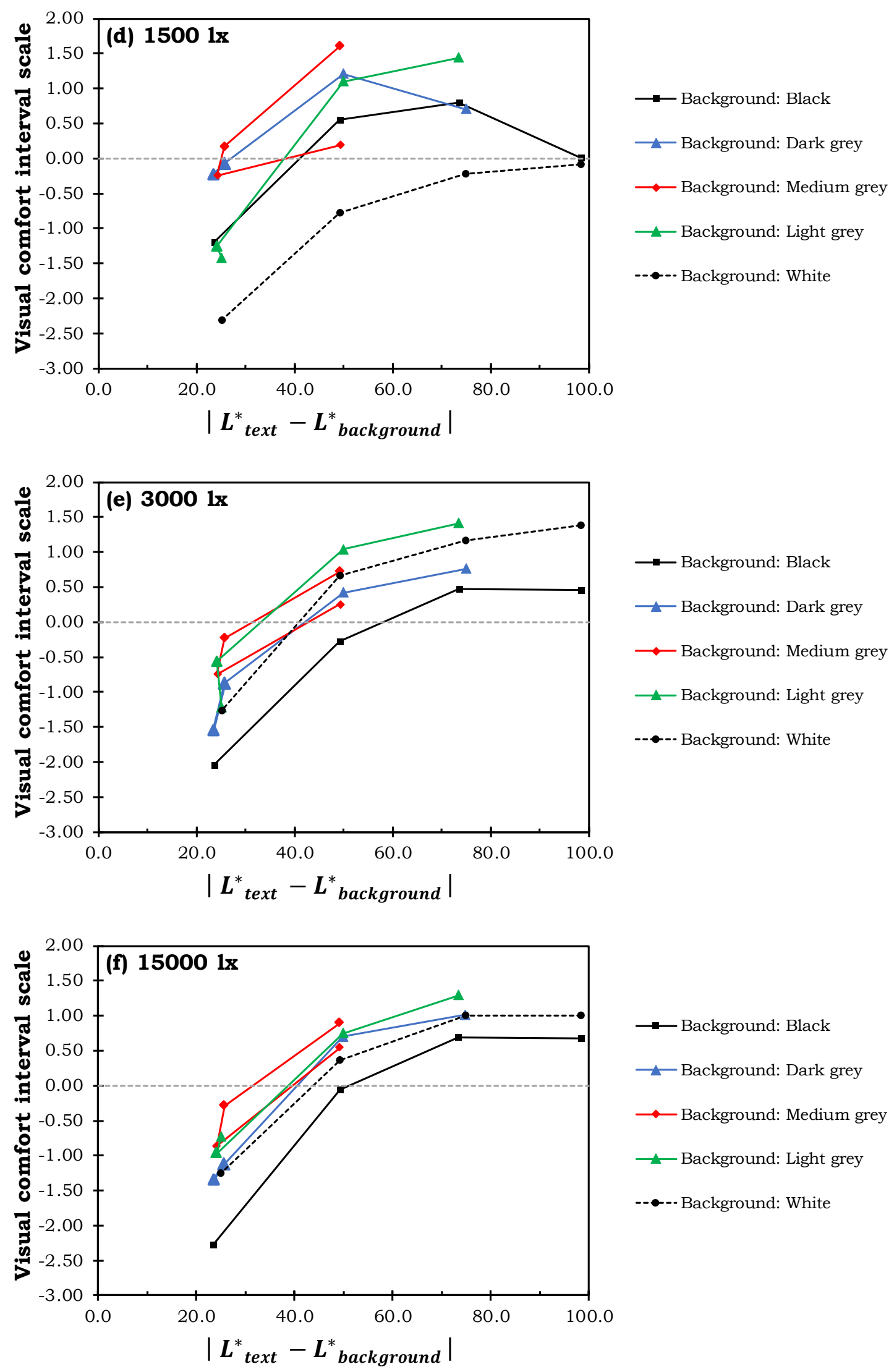

Figure 4 - Visual comfort Interval scale of the 20 text-background combinations evaluated by the observers under each illuminance level (a) Dark; (b) 150 Ix; (c) 300 Ix; (d) 1500 Ix; (e) $3000 \mathrm{Ix}$; (f) $15000 \mathrm{~lx}$ 


\section{Conclusions}

A paired comparison psychophysical experiment was conducted to investigate the visual comfort for e-reading under six ambient illuminance levels, including a dark surround, $150 \mathrm{Ix}$, $300 \mathrm{Ix}, 1500 \mathrm{~lx}, 3000 \mathrm{~lx}$ and outdoor illuminance of $15000 \mathrm{~lx}$. The findings suggest that the observers tended to use different viewing mode for e-reading in interior environments and exterior environments. The experimental results of the study may provide a new guideline for user interface design.

\section{Acknowledgments}

This work was supported in part by The Hong Kong Polytechnic University and the Ministry of Science and Technology, Taiwan (MOST 109-2410-H-263-001).

\section{References}

Chen MT, Lin CC. 2004. Comparison of TFT-LCD and CRT on visual recognition and subjective preference. Int. J. Ind. Ergonom. 34:167-174.

Lin CC, Huang KC. 2006. Effects of ambient illumination and screen luminance combination on character identification performance of desktop TFT-LCD monitors. Int. J. Ind. Ergonom. 36:211-218.

$\mathrm{Na}$, Suk HJ. 2015. Adaptive display luminance for viewing smartphones under low illum inance. Opt Express. 23:16912-16920.

$\mathrm{Na} \mathrm{N}$, Suk HJ. 2016. Adaptive luminance difference between text and background for comfortable reading on a smartphone. Int. J. Ind. Ergonom. 51:68-72.

Huang HP, Wei MC, Ou LC. 2019. Effect of text-background lightness combination on visual comfort for reading on a tablet display under different surrounds. Color Res Appl. 44:5464.

Thurstone LL. 1994. A law of comparative judgment. Psychol. Rev. 101:266-270. 